\title{
DECENTRALIZE ENERGY NETWORK SYSTEM(DEN) IN ASSIMILATION WITH BLOCKCHAIN
}

\author{
Shafali Dhall ${ }^{1}$, Chetanya Ved ${ }^{2}$, Saransh Khetarpaul ${ }^{3}$, Sanjana Deswal ${ }^{4}$, Anmol Dhingra $^{5}$ \\ E-Mail Id: ${ }^{1}$ shafali.dhall@ bharatividyapeeth.edu, ${ }^{2}$ chetanyaved@gmail.com, \\ ${ }^{3}$ saranshkhetarpaul99@gmail.com, ${ }^{4}$ sanjanadeswal2000@gmail.com, ${ }^{5}$ anmoldhingra189@gmail.com \\ Department of Information Technology, Bharati Vidyapeeth's College of Engineering, New Delhi, India
}

Abstract- There is a strong need to establish a chain of energy transfer mechanisms that can lead to a tamper-proof, scalable, and transparent solution. Energy Internet, being a futuristic approach can prevent data tampering and help recording of transactions in a more efficient and a secured way. The clean and direct energy transfer from origin to consumers and its tracking is important to make the transfer of renewable energy a digital and smooth experience. This paper proposes the model of decentralized energy network in assimilation with blockchain. Also, analyzing the applications of blockchain in the energy network, we deliver a novel application of blockchain in decentralized network systems. Further, the paper summarizes the present challenges and their potential future scopes.

Keywords: Smart grid, decentralization, energy, network, blockchain, smart contract.

\section{INTRODUCTION}

\subsection{Blockchain}

Blockchain technology has its own charm by diverting the attention of users towards a series of applications in various fields, since Satoshi Nakamoto proposed the Bitcoin framework[1]. With its advent in 2009, it is gaining importance in all the industries owing to its capacity to change the world in the same way as the internet did back in the 1990's. Moreover, soon after this technology entered to play a major role in the energy sector, it has become one of the popular topics in the field of research. The "distributed ledger technology" paved a new way for the peer to peer network trading of energy which ensures secure transactions without the involvement of a central authority. In a nutshell, blockchain records all energy transactions and once verified, adds them to a public ledger into a block. This process can ensure a greater level of control over energy sources further ensuring a tamper-proof, scalable and an autonomous system without mediation from a third party.

\subsection{Smart Grid}

A smart grid permits systematic communication between an energy consumer and producer. Unlike conventional grid systems where conventional power grid is the nexus of power systems formulated to connect the source of electricity supply with those who exploit electricity, a smart grid empowers both of them to be more versatile and complex in their operational tactics. The foremost Smart Grid systems are interoperability and easily accessible functionalities [2]. The essence of electricity grids is to transmit the energy in a steady and secured manner. Smart grids advance a solution for the fusion of these distributed energy sources to perpetuate the security of the electricity grid. The pivot of smart grids is to provide the ease of local consumption and production by the prosumers and consumers. They both should be capable of trading electricity with each other via peer to peer fashion. To control these transactions engaging within the smart grid in a centralized manner would likely be very costly and would need complex communication infrastructure. As an outcome, it is crystal clear that a decentralized method will be favoured over centralized methods. Inherent properties of a smart grid including its self- healing, modular and distributive generation which also make it a viable choice for the future. Owing to the potential auditable and authorized system, the future of smart energy systems is making access easy for Distributed Energy Resources (DER) [3].

\subsection{Renewable Resources}

Such as solar, wind, and hydro have been present since the beginning of time. Energy generation from these resources is not easy due to the high cost and geographical limitations but if made possible then these sources can produce electricity with less environmental impact than traditional sources. Moreover, with the current rate of depletion of fuel sources and deteriorating environment the use of renewable resources has to be expanded.

\subsection{Non-Renewable Resources}

Since the inception of electricity, non-renewable resources have been credited for the generation of electricity. With the increase in power demands, carbon emissions have become a major concern. Conventional grids using nonrenewable resources are further inaccessible of reaching remote areas. Hence, it becomes a vital requirement to change the patterns of energy generation to make it more scalable, accessible and tamper-proof. Unlike non-renewable resources, generation has started shifting towards a greener and an economical means of energy transfer through the means of renewable resources.

\subsection{Energy Internet}

DOI Number: https://doi.org/10.30780/specialissue-ICACCG2020/021

pg. 100 
ICACCG2020 30-31 July, 2020, Ansal University, Gurgaon, India

International Journal of Technical Research \& Science (Special Issue) ISSN No.:2454-2024 (online)

The increasing demand for reliable, sustainable, renewable and economical energy has been calling for a new paradigm of cyber-physical energy systems, namely the Energy Internet [4]. The Energy Internet can be regarded as an "energy coupling system" which is laid on a simple idea to share economy and resources between parties in a more reliable and transparent manner. Considering the utility advantages of Blockchain paired with the Internet of Things (IoT) [5], the complexities of Energy Internet can be drastically reduced. This paper provides a deep insight into how the paired coupling of Blockchain and IoT can bring a breakthrough in the massive energy sector of the world.

The remaining paper is organised as follows: Section II discusses the problem statements on which the paper has been proposed followed by Section III which briefly sums up the related work. Section IV presents proposed methodology in detail with relevant figures, architecture and components of Energy Internet. Section V provides the use cases of a decentralized energy network system. Features of Decentralized Model of Energy Network are laid out in Section VI. Result Analysis is presented in Section VII followed by the Future Implications and Discussions in Section VIII.

\section{RESEARCH QUESTIONS}

RQ1: What happens when blockchain is assimilated with energy in the field of electric vehicles?

RQ2: How does the energy internet work with a smart grid to control the peak and base of energy flow and carbon footprints?

RQ3: How is the concept of blockchain utilized in the field of Energy Resource Management (ERM)?

RQ4: What is the role of smart contract in microgrid operations?

\section{LITERATURE REVIEW}

In [6] the author has explained about cyberinfrastructure. Cyberinfrastructure was introduced by the NSF in 2003. It comprises of computing frameworks, information storage frameworks, propelled equipment, and information archives, etc., all connected simultaneously by advanced programming and elite frameworks to increase and inquire about efficiency. he author has also explained in detail the four-layer structure given by NSF and that the future goal is to incorporate significant as well as frequently occurring utilities to give a valuable, and empowering system for analysis and disclosure.

In [7] the author has defined cyber-physical systems as a composite framework that profoundly blends networks, computing with material science conditions for attaining ongoing perceiving, data assistance, and dynamic administration. Rather than being a collection of independent devices, a CPS is planned as a system of collaborating components with physical input and yield. CPS can combine the informationalized data into the current framework without upsetting the facilities and computing logic. Simultaneously, any software or structure that has a place within the framework should have the option to progressively join into the framework without being suspended or closed, in order to ensure the framework prerequisites and nature of administration.

In [8] the author has mentioned how the Berkeley structure is constructed using a data-centric energy framework, which is a data overlay on the energy appropriation framework in its different natural indications. By utilizing precharacterized interfaces, energy reserves, providers, intelligent power switches (IPS), and loads can communicate with one another in the subnet. This structure also gives the communication trade that goes with the on-going energy exchanges among provisions and loads.

In [9] the author has proposed an Energy Network Architecture called Future renewable electric energy delivery and management (FREEDM), is a sort of proficient new age environment-friendly energy grid framework, which coordinates exceptionally adaptable and alternate energy generation sources and storage with other accessible power grid frameworks so as to advance green power based industry and society. The FREEDM framework imagined here depends on the wide-scale utilization of renewable energy resources and energy storage devices.

\section{PROPOSED METHODOLOGY}

The decentralized energy network system is the inequitable distribution of Energy and lack of tracking of source and destination for retrieval of required information relies on a base of a middleware to be offered by energy internet which tracks the energy transfer from Green energy producer to a supplier and then from supplier to consumer. As the energy units flow from its source, a decentralized network records each Peer to Peer transaction in a ledger by blockchain. This transaction is stored as a block in the network after validation by one of the adjoining nodes. This energy then gets exchanged as tokens through a set of smart contracts between the two parties. The disruptive powers of Blockchain and automating the entire energy chain not only make the process of energy transfer easy but regular tracking of energy transfer makes the whole process tamper-proof. Considering renewable energy as the future of the energy grids in the near future, it is important to secure the transfer of energy between parties, make them transparent and automatic. Smart contracts being the foundation of this automation, it is of utmost importance to ensure a trustable system that can prevent any kind of tampering with the delivery system. Our proposed method will serve as a backbone to the energy chain network in each peer to peer network setup. The objective of setting up an efficient information interaction between each unit that is a part of the energy chain establishing an efficient transmission can potentially be achieved by the benefits of Blockchain and Internet of Energy where each network has the power to become an energy generator and seller as shown in fig. 4.1. This will hence lead to a credible, equitably managed, sustainable, and cost-effective system.

DOI Number: https://doi.org/10.30780/specialissue-ICACCG2020/021

pg. 101

Paper Id: IJTRS-ICACCG2020-021

@ 2017, IJTRS All Right Reserved, www.ijtrs.com 
ICACCG2020 30-31 July, 2020, Ansal University, Gurgaon, India

International Journal of Technical Research \& Science (Special Issue) ISSN No.:2454-2024 (online)

Through the utilization of intelligent features such as descriptive interfaces that cover the technology involved and intelligent edge switches, the Internet has gone through tremendous improvements. The sole purpose of these switches is to control access of the user. This benefits the internet in enabling access rights for devices but decreases the capacity of packet forwarding per unit time. As for energy routers, the hierarchy mechanism of the routers should always be designed such that it fulfils different requirements of energy routing like backbone switches do on the internet. Operations are then adjusted to intelligent terminals according to the local power supply situation of the infrastructure.

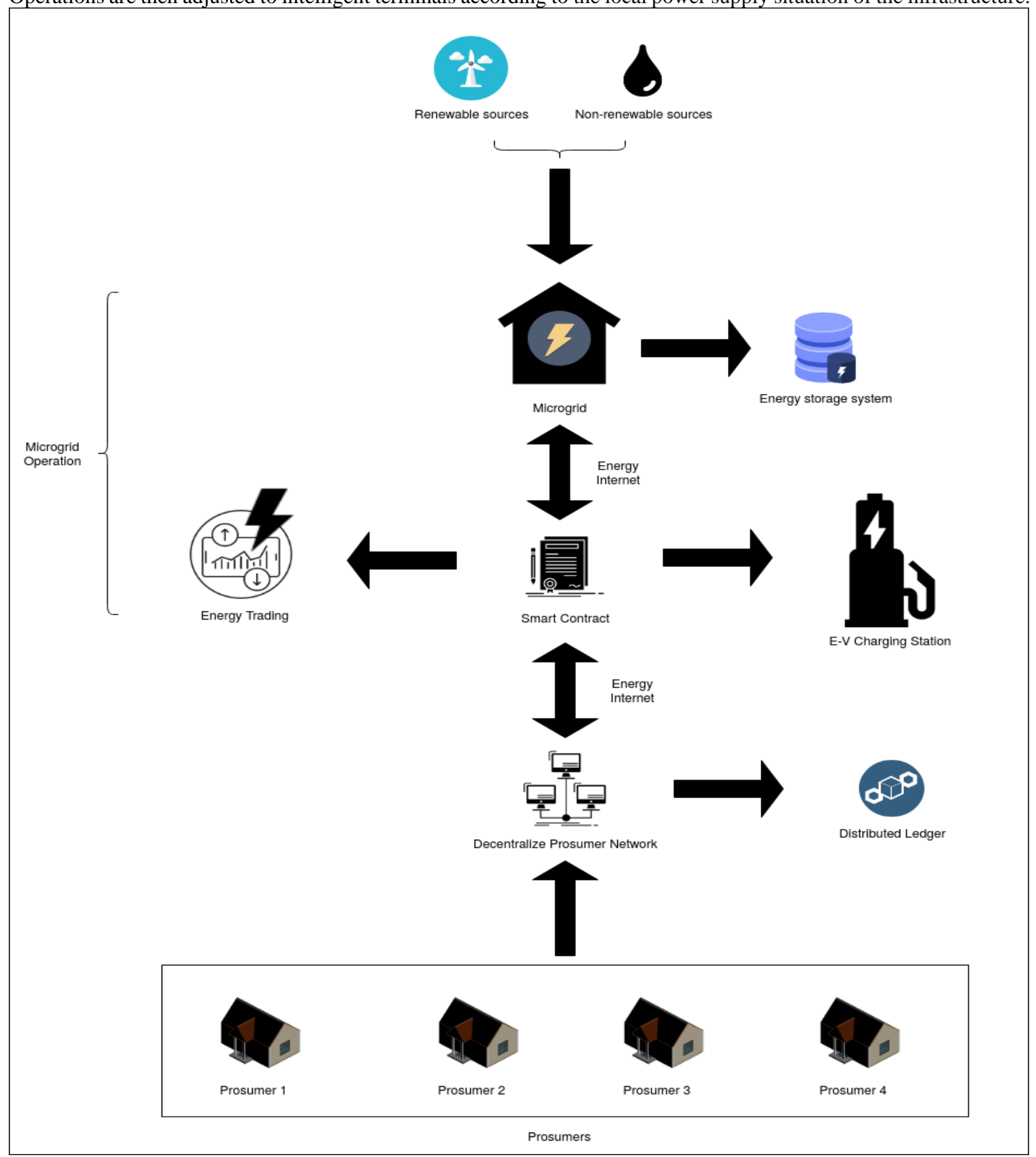

Fig. 4.1 Model of Decentralize Energy Network (DEN)

\subsection{Architecture}

Many small microgrids and power stations collaborate to form a giant Energy generation network. The dynamic bus provided by introducing the concept of Energy Internet plays the role of "dynamic" information storage. The word dynamic data unit implies the flexible nature of data bus to expand and contract according to the incoming energy and requirements. This further allows networks to avoid conventional update cycle fashions and hence dependency on non-renewable resources for electricity generation. Further, Energy routers play a major role in the transmission of accumulated energy. Also, the contemplation between the generation and load is broken down by the primary utility distribution of energy. As soon as this energy reaches its potential consumers and business units, smart contracts help 
to retransmit and utilize this energy for individual and collective purposes. A smart contract paired with Blockchain is responsible to maintain a ledger consisting of Energy transfer records. Role of Energy Internet becomes crucial in the whole process of generation of energy through microgrids to the utilization of energy by end-users. The effectual and bifacial flow of power can be made through the assimilation of information with energy [10].

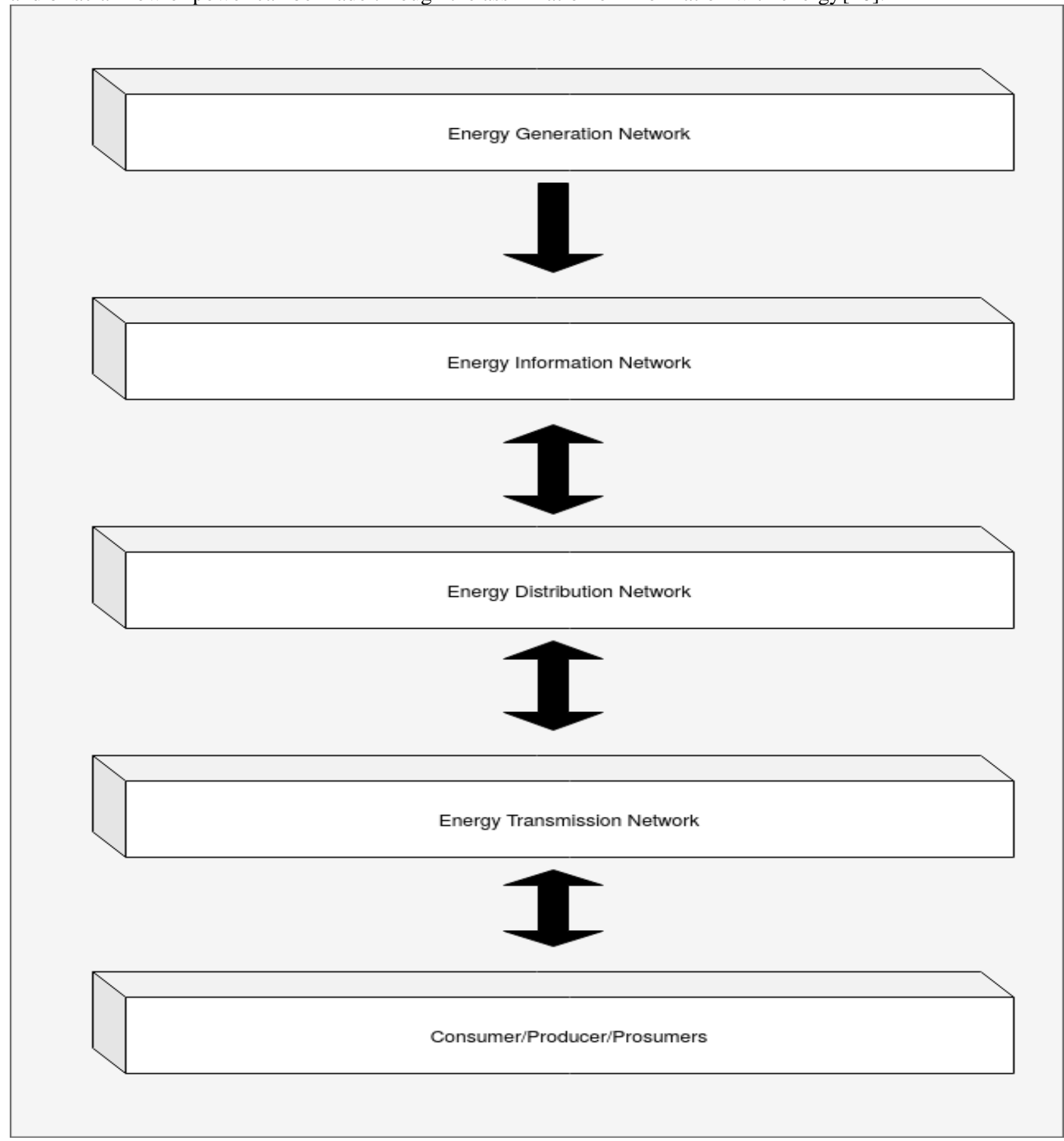

Fig. 4.2 Five-Tier Architecture of Decentralize Energy Network

\subsection{Components Used}

Energy Network consists of three major parts: generation, transmission, and distribution. It has many control nodes and a router that can be utilized for different degrees of control which further helps the peer-2-peer network of energy to execute smoothly. This network of energy is based on the principle of decentralization which means that every node in the network has access to all energy transactions. Here, data is continually generated, duplicated, and stored away. Nonetheless, the Energy Network cannot regenerate energy. Therefore, the supplied energy needs to be adjusted by demand. Following are some essential hardware components of the energy network-

\subsubsection{Energy Router}

The energy router is a fundamental component of the Energy Network [11]. The energy router comprises a solid-state transformer, a disseminated network intelligent control system, and a communication unit. The router can receive, process, and transmit the data of the network. The router is equipped for expanding the network's reliability, 
ICACCG2020 30-31 July, 2020, Ansal University, Gurgaon, India

International Journal of Technical Research \& Science (Special Issue) ISSN No.:2454-2024 (online)

productivity, and security of the power network, and in this way, can upgrade the energy utilization by adjusting the supply and demand. On the off chance if the supply of energy surpasses the demand, at that point the energy router moves the surplus energy to the utility grid [12].

\subsubsection{Energy Storage Systems}

The storage systems in the energy network can remarkably improve grid productivity, dependability, and reliability. The detailed utilization and application of the storage systems have been explored in [13-15]. A storage device can decrease the pressure of a grid and store energy for some time in the future, consequently guaranteeing the smooth flow of power [16]. The storage system can improve power quality [17] and tackle the issue of voltage changing rapidly [18]. Ordinarily utilized storage devices are batteries, supercapacitors, fuel cells, flywheel, packed air, and pumped hydro.

\subsubsection{Energy Generation Sources}

Energy can be generated both from renewable and non-renewable resources, both of which are integrated into the main grid. For sustainable development, wellbeing, and environment, the network of energy comprises different interconnected sustainable sources, while the non-renewable is kept as a backup in case renewable sources cannot generate energy due to environmental conditions. Renewable advances with energy storage frameworks have become the generally acknowledged answers for accomplishing this steady and reliable green environment [19], [20-22]. The integration of renewable advancements requires power converter topologies and control strategies.

\subsubsection{Microgrid}

A microgrid is connected to almost every other entity in the network. It is connected to the energy generation systems through the energy router which controls the flow of electricity and establishes a secure data transfer and communication unit. Next it is connected to the energy internet to enable transparent and flawless transfer of energy and data. Further it is connected to energy storage devices in case renewable sources cannot generate electricity due to environmental conditions and other micro grids to expand the network and form a bigger smart grid. Microgrid is the part that facilitates functions such as energy trading and EV charging stations.

\subsubsection{Energy Internet}

The energy internet can be expounded as an internet-based wide area network (WAN) for energy fusion and information. It is a broad network that systematically provides electricity throughout. EI not only admits the infrastructure but also places the higher demand on the intelligent system of energy. On the one side, due to the lofty seepage of renewable energy resources in EI, their fluctuating output will intensify the load volatility of grid; whereas on the other side, the EI molds the centralized infrastructure into the distributed energy management, in which the distributed microgrids with varied scales are liable for controlling the densely expanded demand units.

\subsubsection{Plug and Play Appliance Integration}

The plug-and-play interface of the energy network encourages the association of sustainable sources and storage devices. The plug-and-play interface may have distinctive interfacing strategies (AC/DC) given that either AC or DC microgrid (MG) can associate through its interface. The reconciliation of home appliances or any other load is additionally significant as the demand and supply should be adjusted for the effective activity of the network of energy. Here, propelled studies on utilizing home appliances can diminish the demand for power and greenhouse gas discharge.

\section{USE-CASE OF DECENTRALIZE ENERGY NETWORK}

Regularly clients are seen to rely on a central authority because of which there is a high danger of security breach. In the event of decentralization, various hubs deal with the system and there is no further reliance on the focal power. Accordingly, the information is made sure about at further degree and there is no danger of loss of information. Much of the time, the system gets fizzled and subsequently entire information gets smashed, which is a significant issue. To illuminate this, the procedure of decentralization raised advanced monetary standards as digital currencies which diminish the opportunity of disappointment of the systems. Further, the energy can be conveyed among the companions or charging stations by means of the procedure of decentralization going about as energy trading and these tasks are conveyed under microgrids.

The main segments where the applications of decentralization are broadly applied in the energy sector according to our model are listed below.

\subsection{Energy Trading}

According to the model, the customer can impart the power to its peers and through DLT, the prosumer can think about the utilization of power by the nodes. Additionally, the prosumer can know which node is missing with the lack of energy and which node has surplus amounts. This entire procedure can be characterized as energy Trading. 


\subsection{Electricity Charging Vehicles}

In the event that, when there is no prosumer confronting the issue of absence of power, at that point the framework can send the excess sum in the E-V stations to charge the vehicles and they can pay for that. The assimilation of blockchain with the process of EV charging makes sure the best price and the location for the users of EV while ensuring the privacy and the security of the entire system via decentralization.

\subsection{Microgrid Operations}

Management of microgrids has become an important aspect particularly with the assimilation of various distributed energy resources (DERs). The requirement for optimized operation and demand-based management of microgrids is the main focus nowadays for the researchers. Blockchain technology is included in this particular area for the possible profits.The scheduling mechanism of DERs is based on blockchain technology. The usage of blockchain in this field furnishes a trustworthy plan so that all DERs are secured and trusted.

\section{FEATURES OF DECENTRALIZE ENERGY NETWORK}

\subsection{Upgraded Customer Experience}

The most significant component of the energy network is that energy units can be packed and moved securely, regardless of when and where the clients are in need [23]. Clients can take an interest in each productive energy utilization interface with the goal that clients can pick the sources of energy use as well as to take part during the energy generation and sales, further understanding the change from consumers to producers. This implies the energy network understands clients' experience, particularly stressing on fulfilling the demands of clients.

\subsection{Increased Transparency}

Transparency is another key component of the Energy Network. An energy network framework can combine open energy, all equipment, and frameworks of open access, open energy markets, and exchanging platforms [24]. The energy network framework is devoted to building an open environmental framework; it can draw in more energy for another plan of action, understanding the organic combination of disseminated energy and the smart grid.

\subsection{Improved Utilization}

Communication in energy networks provides increasingly explicit instantaneous information on dispersed energy production, power transmission, and utilization. This encourages the utility administrators to enhance their decision advances by figuring out which part is conceivable to come up short and deciding the substitution strategy on the Internet. [25].

\subsection{Efficiency in Energy Distribution}

The efficiency of energy utilization can be significantly improved by actualizing the open interrelationship, planning enhancement of energy, and presenting a setting for the extensive advancement of energy, energy utilization, and sharing [26].

\subsection{Growth in the Energy Market}

The newly emerged market breaches all boundaries of the industry, giving the least expensive and advantageous platform for an enormous number of members and clients. Targeted at understanding success in the circumstances, stimulating the eagerness of advancement, and at last giving the constant power for the new energy transformation [27].

\subsection{Energy Conservation}

The energy network encourages the high infiltration of sustainable power source access and utilization. Through an assortment of corresponding energy coupling, a wide range of energy stockpiling applications, the demand side response (DMS), and so on., the high infiltration of sustainable power source access and utilization can be strongly upheld [28].

\subsection{Real-time Data Management}

Unlike conventional grid systems that work on a static update cycle to meet the demands of consumers, Energy Internet has evolved as a unique methodology to minimize the use of conventional power sources and process real-time data to meet the requirements of consumers by maintaining an ever-growing data bus.

\section{RESULT ANALYSIS}

This model of the decentralized energy network aims to provide electricity to the deprived section of the population. Through this model, we are providing the capability to the consumer to become a producer as well as a consumer which turns out to be a prosumer. Adoption of this model will save a lot of excessive units of electricity. The motive of designing this type of decentralized energy network is to make judicial usage and distribution of energy among different nodes. Energy trading among peer-2-peer connected nodes will make this model more financially feasible. A distributed ledger will maintain transparency among trustable and untrustable parties. The smart contract will manage to switch the process between energy trading among peer-2-peer networks or trading between prosumer to 
ICACCG2020 30-31 July, 2020, Ansal University, Gurgaon, India

International Journal of Technical Research \& Science (Special Issue) ISSN No.:2454-2024 (online)

charging stations, which will lead to efficient utilisation of excessively produced units of electricity and it will be incentivised to prosumer in terms of energy tokens which can be monetarily redeemed. This will develop an incentivised energy exchange ecosystem. It will be an economical and environmental-friendly approach to solve energy-related crises and issues.

\section{DISCUSSION, IMPLICATION AND FUTURE SCOPE}

Throughout the paper, we have explored the present scenario of decentralized energy networks in the generation of electricity through smart grids. Since the power transfer through traditional grids is carbon-intensive and expensive as a matter of fact, more than $13 \%$ of the world's population is out of the range of electricity. Hence, it is an acute need of replacing the conventional grids with smart grid systems that run autonomously by a set of predefined rules. This will help meet the local demands of the households and can reclaim the poles and wires into "smart neighbourhoods". Making a prosumer [30] the core entity, it brings the overall energy cost down.

Introducing the concept of "Energy crowdfunding" in a decentralized Energy network will serve as a future scope in our system. Another improvement in the generation of Green Energy can be the idea of Energy Crowdfunding. Through this unique idea, it is aimed to solve the problems of the generation of cleaner energy even in areas that are unable to generate green energy. It uses a platform where people can become collaborators on projects which generate clean energy. Each of these projects is proposed by a group of developers asking for contributions to sustain its viability. After the extensive review of each process and viability check of each process, people can now choose to be the contributors to the project sustaining the criteria. This contribution is done in the form of an exchange of tokens against the amount raised for funding. These tokens start generating a stream of energy tokens that are transferred between the parties as soon as the plant starts generating energy. Any type of customer irrespective of living conditions, can contribute and get benefitted by such a system through lower costs and increased economy. This is even better than

traditional systems that buy electricity at wholesale prices from plants and sell them with high margins to end customers. Along with this, the model contains various challenges such as : In the facet of data, the concern should be on the techniques to avoid the interruption in pernicious conduct, the transmission of data, and capacity of security insurance. Likewise, more consideration is to be merited by the transmission of energy as well as data. Another challenge can be the Unwavering quality where the objective of decentralized energy networks is to use energy technology and Internet information technology to give prime concern to renewable energy sources. The success of an energy network is based on how to utilize distributed energy efficiently, and impart the supply of stable energy. Concluding with the last challenge as setting up data collaboration between every arrangement of transmission of energy and its effectiveness has become a difficulty which should be illuminated $[31,29]$

\section{REFERENCES}

[1] Tianyu Yang, Qinglai Guo, Xue Tai, Hongbin Sun, Boming Zhang, Wenlu Zhao, Chenhui Lin, "Applying Blockchain Technology to Decentralized Operations in Future Energy Internet." in 2017 IEEE Conference on Energy Internet and Energy System Integration (EI2), 1-5, 2017

[2] Nicola Bui, Angelo Castellani, Paolo Casari, Michele Zorzi, "The Internet Of Energy: A web-Enabled Smart Grid system", IEEE Network 26 (4), 39-45, 2012

[3] Michael Mylrea, Sri Nikhil Gupta Gourisetti, "Blockchain for smart grid resilience: Exchanging distributed energy at speed, scale and security", 2017 Resilience Week (RWS), 18-23, 2017

[4] Xinghuo Yu, Yusheng Xue, "Smart Grids: A cyber-Physical Systems Perspective" in Proceedings of the IEEE 104 (5), 1058-1070, 2016

[5] Konstantinos Christidis, Michael Devetsikiotis, "Blockchains and smart contracts for the internet of things" Ieee Access 4, 2292-2303, 2016

[6] J. Cao (Eds.), "Cyberinfrastructure Technologies and Applications”, Nova Science Publishing, 2012

[7] Edward Lee, "Cyber Physical Systems : Design Challenges", University of California, Berkely, Technical Report No. UCB/EECS-2008-8

[8] Randy H. Katz, David E. Culler, Seth Sanders, Sara Alspaugh, Yanpei Chen, “ An information-centric energy infrastructure : The Berkeley View", (2011) 7-22:8-24

[9] A. Huang, "FREEDM system - A vision for the future grid",in Proc. IEEE Power Energy Soc. Gen. Meeting, Jul. 2010, pp. 1-4

[10] Junwei Cao and Mingbo Yang, "Energy Internet-Towards Smart Grid2.0", in 2013 Fourth international conference on networking and distributed computing.

[11] K. Wang, H. Li, Y. Feng, and G. Tian, "Big data analytics for system stability evaluation strategy in the energy Internet," IEEE Trans. Ind. Informat., vol. 13, no. 4, pp. 1969-1978, Aug. 2017

[12] Y. Xu, J. Zhang, W. Wang, A. Juneja, and S. Bhattacharya, "Energy router: Architectures and functionalities toward energy Internet," in Proc. IEEE Int. Conf. Smart Grid Commun. (SmartGridComm), Oct. 2011, pp. 3136.

[13] M. A. Hannan, M. M. Hoque, A. Mohamed, and A. Ayob, "Review of energy storage systems for electric vehicle applications: Issues and challenges,” Renew. Sustain. Energy Rev., vol. 69, pp. 771-789, Mar. 2017. 
ICACCG2020 30-31 July, 2020, Ansal University, Gurgaon, India

International Journal of Technical Research \& Science (Special Issue) ISSN No.:2454-2024 (online)

[14] O. Palizban and K. Kauhaniemi, "Energy storage systems in modern grids-Matrix of technologies and applications,', J. Energy Storage, vol. 6, pp. 248-259, May 2016.

[15] R. Xiong, J. Cao, Q. Yu, H. He, and F. Sun, 'Critical review on the battery state of charge estimation methods for electric vehicles,'” IEEE Access, vol. 6, pp. 1832-1843, 2017.

[16] A. S. Subburaj, B. N. Pushpakaran, and S. B. Bayne, "Overview of grid-connected renewable energy-based battery projects in the USA,', Renew. Sustain. Energy Rev., vol. 45, pp. 219-234, May 2015.

[17] J. Mundackal, A. C. Varghese, P. Sreekala, and V. Reshmi, "Grid power quality improvement and battery energy storage in wind energy systems,' in Proc. Annu. Int. Conf. Emerg. Res. Areas, Int. Conf. Microelectron., Commun. Renew. Energy, Jun. 2013, pp. 1-6.

[18] G. Huff et al., “DOE/EPRI 2013 electricity storage handbook in collaboration with NRECA,'” Tech. Rep., Jul. 2013 , p. 340.

[19] J. G. J. Olivier, G. Janssens-Maenhout, M. Muntean, and J. A. H. W. Peters, "Trends in global CO2 emissions: 2016 report,'’ PBL Netherlands Environmental Assessment Agency, The Netherlands, Tech. Rep., 2016 , p. 86.

[20] A. Kostevšek, J. J. Klemě̌, P. S. Varbanov, L. Čuček, and J. Petek, "Sustainability assessment of the locally integrated energy sectors for a Slovenian municipality,' J. Cleaner Prod., vol. 88, pp. 83-89, Feb. 2015.

[21] 2014CAR: United States Climate Action Report 2014, Six Nat. Commun. USA United Nations Framework Convention Climate Change, U.S. Dept. State, Washington, DC, USA, 2014.

[22] G. Boukettaya and L. Krichen, "A dynamic power management strategy of a grid-connected hybrid generation system using wind, photovoltaic and flywheel energy storage system in residential applications,' Energy, vol. 71, pp. 148-159, Jul. 2014.

[23] S. Kumar and S. Sachdev, "Amalgamation of Internet for supervisory control and data acquisition (SCADA) encompassing security issues," in Proc. IEEE Data Storage Data Eng., Feb. 2010, pp. 1-4.

[24] R. Davies, "Hydro one's smart meter initiative paves way for defining the smart grid of the future," in Proc. IEEE Power Energy Soc. Gen. Meeting, Jul. 2009, pp. 1-2.

[25] V. Altmann, J. Skodzik, F. Golatowski, and D. Timmermann, "Investigation of the use of embedded web services in smart metering applications," in Proc. 38th Annu. Conf. IEEE Ind. Electron. Soc., Oct. 2012, pp. 6172-6177.

[26] C. Wei and Y. Li, "Design of energy consumption monitoring and energy-saving management system of intelligent building based on the Internet of Things," in Proc. IEEE Electron., Commun. Control, Sep. 2011 ,pp. 3650-3652.

[27] Y. Suh, J. Choi, C. Seo, and Y. Shin, “A study on energy savings potential of data network equipment for a green Internet," in Proc. IEEE Adv. Commun. Technol., Feb. 2014, pp. 1145-1151.

[28] I. Ciuciu, R. Meersman, and T. Dilon, "Social network of smart metered homes and SMEs for grid-based renewable energy exchange," in Proc. IEEE Digit. Ecosystems Technol., Jun. 2012, pp. 1-6.

[29] Wang, K., Yu, J., Yu, Y., Qian, Y., Zeng, D., Guo, S., .. Wu, J. (2017). A Survey on Energy Internet: Architecture, Approach, and Emerging Technologies. IEEE Systems Journal, 1-14. doi:10.1109/jsyst.2016.2639820

[30] Santiago Grijalva, Muhammad Umer Tariq, " Prosumer- based smart grid architecture enables a flat, sustainable electricity energy", ISGT 2011, 1-6, 2011

[31] National Renewable Energy Laboratory (NREL). 2012. Renewable Electricity Futures Study. 